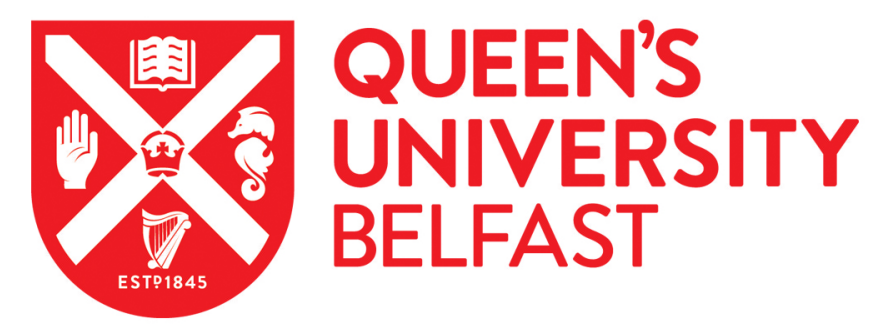

\title{
Performance Analysis of OTFS-based Uplink Massive MIMO with ZF Receivers
}

Feng, J., Ngo, H-Q., Flanagan, M. F., \& Matthaiou, M. (2021). Performance Analysis of OTFS-based Uplink Massive MIMO with ZF Receivers. In 2021 IEEE International Conference on Communications ( IEEE International Conference on Communications Workshops (ICC Workshops): Proceedings). Institute of Electrical and Electronics Engineers Inc.. https://doi.org/10.1109/ICCWorkshops50388.2021.9473699

\section{Published in:}

2021 IEEE International Conference on Communications

\section{Document Version:}

Peer reviewed version

\section{Queen's University Belfast - Research Portal:}

Link to publication record in Queen's University Belfast Research Portal

\section{Publisher rights}

(c) 2021 IEEE.

This work is made available online in accordance with the publisher's policies. Please refer to any applicable terms of use of the publisher.

\section{General rights}

Copyright for the publications made accessible via the Queen's University Belfast Research Portal is retained by the author(s) and / or other copyright owners and it is a condition of accessing these publications that users recognise and abide by the legal requirements associated with these rights.

Take down policy

The Research Portal is Queen's institutional repository that provides access to Queen's research output. Every effort has been made to ensure that content in the Research Portal does not infringe any person's rights, or applicable UK laws. If you discover content in the Research Portal that you believe breaches copyright or violates any law, please contact openaccess@qub.ac.uk. 


\title{
Performance Analysis of OTFS-based Uplink Massive MIMO with ZF Receivers
}

\author{
Junjuan Feng*, Hien Quoc Ngo*, Mark F. Flanagan ${ }^{\dagger}$, and Michail Matthaiou* \\ *Institute of Electronics, Communications and Information Technology (ECIT), Queen's University Belfast, U.K. \\ ${ }^{\dagger}$ School of Electrical and Electronic Engineering, University College Dublin, Belfield, Dublin, Ireland \\ Email:\{junjuan.feng, hien.ngo\}@qub.ac.uk, mark.flanagan@ieee.org, m.matthaiou@qub.ac.uk
}

\begin{abstract}
This paper considers an orthogonal time frequency space (OTFS)-based uplink massive multiple-input-multipleoutput (MIMO) system, in which all users transmit symbols to a base station (BS) that is equipped with an one-dimensional uniform linear array (ULA) in the delay-Doppler domain. The zero-forcing (ZF) decoding scheme is applied at the BS. Different from most existing OTFS works, this paper mainly focuses on the performance analysis. Furthermore, in contrast to the existing massive MIMO works, which assume favorable propagation (FP) conditions, here, we investigate the low but non-zero probability scenario that massive MIMO fails in providing FP. This results in a scenario where the channel matrix cannot be diagonalized with massive MIMO. Thus, to facilitate the performance analysis, a low-complexity approximation of the inverse of the Gram matrix, i.e., the Neumann series expansion, is presented. With this, a simple signal-to-noise-ratio (SNR) approximation is firstly derived, which illuminates the impact of the OTFS system parameters. Moreover, by exploiting the properties of circulant matrices, a compact expression for the OTFS multi-path correlation matrix (OTFS-MPCM) is derived.
\end{abstract}

Index Terms-Angular spread, Massive MIMO, Neumann series, OTFS, ULA

\section{INTRODUCTION}

Future beyond-5G wireless communication systems will be expected to achieve reliable transmission at high data rates in rapidly varying channel conditions, which motivates research efforts on communications over high-mobility and delay spread wireless channels [1]. Such channels are highly doubly-dispersive in the time-frequency domain, due to the time delay caused by the multi-path propagation and the rapid frequency dispersion caused by high Doppler shift. The celebrated orthogonal frequency division multiplexing (OFDM) technique has been used to mitigate this effect by reducing inter-user interference. However, it entails the issue of inter-carrier interference, which can substantially degrade the system performance [2].

Recently, a new modulation scheme, known as orthogonal time frequency space (OTFS), was proposed in [3]. The key idea in OTFS is that the information symbols are embedded in the delay-Doppler domain rather than in the time-frequency domain as in OFDM. This new modulation scheme transforms a time-varying multi-path channel in the time-frequency domain into a 2-dimensional (2D) timeinvariant channel in the delay-Doppler domain, such that each information symbol experiences a near-constant channel gain, which can greatly help to reduce resources used for channel

This work was supported by research grants from the Department for the Economy Northern Ireland, and from Science Foundation Ireland (grant number 17/US/3445), under the US-Ireland R\&D Partnership Programme. estimation and also simplify the system design [4]. OTFS has been proved to exhibit lower error rate than OFDM [3].

Recognizing the significant benefits of OTFS modulation, many research works have recently appeared in the literature. Among these, [5] proposed low-complexity minimum mean square error (MMSE) and ZF equalizers for the single-inputsingle-output (SISO) OTFS networks and exploited a decomposition of the associated doubly-block circulant matrices; also, [6] exploited the sparse structure of the OTFS channel matrices. OTFS modulation was extended to the MIMO case in [7], which presented a channel estimation scheme and a message-passing based signal detection algorithm. Following this, an OTFS-based uplink multiple-access network was studied in [4], which proposed an uplink multiple-access method to avoid multi-user interference in OTFS. In order to incorporate OTFS modulation into the massive MIMO space, a novel uplink and downlink massive MIMO channel estimation algorithm was presented in [8] and [9] to reduce the training overhead in a high-mobility scenario, respectively. Further, a downlink multi-user massive MIMO system was considered in [10], which proposed a novel multi-user precoder and low-complexity detector.

Most of the above-mentioned works focused on channel estimation or low-complexity precoder/detector design. To the best of our knowledge, little research work exists regarding the performance analysis of OTFS modulation. Thus, this paper will focus on the performance analysis of an uplink OTFS multi-user massive MIMO system. Recall now that massive MIMO with linear signal processing is capable of achieving higher spectral efficiency by leveraging favorable propagation (FP) and channel hardening $(\mathrm{CH})$ [11]. However, in practice, whether or not FP can be obtained is highly dependent on the exact locations of the users and the BS, as has been shown in [12] and [13]. This means that we cannot always obtain orthogonal channels among users with massive MIMO, which renders the performance analysis and system design problematic when some linear signal processing schemes, such as ZF and MMSE, are applied. Against this background, the main contributions of this paper are listed as follows:

1) We consider an OTFS-based uplink multi-user massive MIMO network, where the ZF scheme is applied at the $\mathrm{BS}$ to decode the received symbols. A rigorous performance analysis of this system is given.

2) In contrast to most existing massive MIMO works, we consider the low-probability scenario where massive MIMO fails to offer FP, i.e., fails to make the uplink 


$$
y_{p}[k, l]=\frac{1}{M N} \sum_{q=1}^{K_{u}} \sum_{k^{\prime}=0}^{M-1} \sum_{l^{\prime}=0}^{N-1}\left[\iint h_{q, p}(\tau, \nu) w\left(\frac{k-k^{\prime}}{M \Delta f}-\tau, \frac{l-l^{\prime}}{N T}-\nu\right) e^{-j 2 \pi \nu \tau} d \nu d \tau\right] x_{q}\left[k^{\prime}, l^{\prime}\right]+\omega_{p}[k, l]
$$

channels orthogonal. When this occurs, the uplink OTFS channels cannot be diagonalized. In this case, we apply a low-complexity approximation of the inverse of the Gram matrix, the Neumann series (NS) expansion. This makes our results for OTFS simpler and amenable to further mathematical manipulations.

3) By harnessing the properties of circulant matrices that each circulant matrix can be expressed as a polynomial function of the primary permutation matrix, the OTFSbased multi-path correlation matrix (OTFS-MPCM) is derived. It is shown that the multi-path delay and Doppler shift rotate the phase of each entry of the OTFS-MPCM, while the multi-path direction of arrival not only affects the phase of each entry but also the corresponding magnitude.

\section{System MODEL}

In this section, we provide the details of our model for OTFS-based uplink MU-MIMO transmission, in which a common BS serves $K_{u}$ single-antenna users simultaneously. The BS is equipped with a one-dimensional $N_{b}$-element uniform linear array (ULA), where the distance between any two adjacent antennas is denoted by $d_{a}$.

In OTFS, the data symbols transmitted by each user are modulated in the delay-Doppler domain. We assume that the total $T$ seconds in the delay domain are divided into $M$ equal blocks, while the total $\Delta f=\frac{1}{T} \mathrm{~Hz}$ in the Doppler domain are divided into $N$ equal blocks. The maximum possible delay spread $\tau_{\max } \leq T$, while the maximum possible Doppler shift $\nu_{\max } \leq \Delta f$. Let $x_{q}[k, l] \in \mathbb{C}$, for $q=1, \ldots, K_{u}$, be the data symbol transmitted by the $q$-th user on the $(k, l)$-th delayDoppler sub-block, which is allocated over the delay-Doppler lattice

$$
\Lambda=\left\{\left(\frac{k}{M \Delta f}, \frac{l}{T N}\right) \mid \begin{array}{c}
k=0, \ldots, M-1 \\
l=0, \ldots, N-1
\end{array}\right\}
$$

We assume that the transmit and receive pulse shaping functions are respectively orthogonal as well as biorthogonal, and that rectangular window functions in the time-frequency domain are used at both the users' side and the BS side. After the OTFS modulation at the users' side and demodulation at the BS side, the details of which can be found in [3], the final received signal at the $p$-th antenna of the $\mathrm{BS}$ is expressed as (2) at the top of this page, where $\omega_{p}[k, l]$ is the additive white Gaussian noise (AWGN) at the $p$-th antenna of the BS with the distribution $\omega_{p}[k, l] \sim \mathcal{C N}\left(0, \sigma^{2}\right)$. The scalar $h_{q, p}(\tau, \nu)$ is the channel response in the delay-Doppler domain from the $q$-th user to the $p$-th antenna of the BS at delay $\tau$ and Doppler shift $\nu$, which has bounded support, i.e.,

$$
h_{q, p}(\tau, \nu)=0, \quad|\tau|>\tau_{\max } \quad \text { or }|\nu|>\nu_{\max } .
$$

The window function $w(\tau, \nu)$ is given by

$$
w(\tau, \nu)=\sum_{m=0}^{M-1} \sum_{n=0}^{N-1} e^{-j 2 \pi(\nu n T-\tau m \Delta f)} .
$$

Now let $\mathbf{h}_{q}(\tau, \nu)=\left(h_{q, 1}(\tau, \nu), \quad \ldots, \quad h_{q, N_{b}}(\tau, \nu)\right)^{T} \in$ $\mathbb{C}^{N_{b} \times 1}$ denote the uplink channel vector from the $q$-th user to the BS in the delay-Doppler domain, where $(\cdot)^{T}$ denotes the transpose of vectors or matrices. Since a ULA is considered at the BS, the channel vector $\mathbf{h}_{q}(\tau, \nu)$ can be written as $[14$, Example 1.8], [15]

$$
\mathbf{h}_{q}(\tau, \nu)=\sum_{i=1}^{M_{q}} h_{q, i} \mathbf{a}\left(\theta_{q, i}\right) \delta\left(\tau-\tau_{q, i}\right) \delta\left(\nu-\nu_{q, i}\right),
$$

where $M_{q}$ denotes the number of paths from the $q$-th user to the $\mathrm{BS}$. The vector $\mathbf{a}\left(\theta_{q, i}\right) \in \mathcal{C}^{N_{b} \times 1}$ is the spatial array steering vector, which is defined as

$\mathbf{a}\left(\theta_{q, i}\right)=\frac{1}{\sqrt{N_{b}}}\left[1, e^{j 2 \pi \frac{d_{a} \sin \left(\theta_{q, i}\right)}{\lambda}}, \ldots, e^{j 2 \pi\left(N_{b}-1\right) \frac{d_{a} \sin \left(\theta_{q, i}\right)}{\lambda}}\right]^{T}$.

In (5) and (6), the notations $\tau_{q, i}, \nu_{q, i}$, and $h_{q, i}$ denote the delay, Doppler shift, and the channel gain, respectively, of the $i$-th path of the $q$-th user. The random variables $h_{q, i}$ for different $(q, i)$ are independent and have distribution $h_{q, i} \sim$ $\mathcal{C N}\left(0, \beta_{q, i}^{2}\right)$ [11]. The parameter $\theta_{q, i} \in\left[-\frac{\pi}{2}, \frac{\pi}{2}\right)$ denotes the direction of arrival (DoA) for the $i$-th path of the $q$-th user and $\lambda$ denotes the signal wavelength. The delay and Doppler shift for the $i$-th path are given by

$$
\tau_{q, i}=\frac{k_{q, i}}{M \Delta f}, \quad \nu_{q, i}=\frac{l_{q, i}}{N T} .
$$

For ease of derivations, we assume that the coefficients $k_{q, i}$ and $l_{q, i}$ are integers (this is an accurate assumption if $M$ and $N$ are sufficiently large), and that they satisfy $k_{q, i} \in[0, M-$ 1] and $l_{q, i} \in[0, N-1]$, respectively [16]. We also assume perfect knowledge of the parameters $\left\{h_{q, i}, l_{q, i}, k_{q, i}\right\}_{i=1 \ldots, M_{q}}^{q=1, \ldots, K_{u}}$ at the BS.

Let $(\cdot)_{N}$ denote the modulo $N$ operation. Substituting $w(\tau, \nu)$ in (4) and the $p$-th element $h_{q, p}(\tau, \nu)$ in (5) into (2), the final OTFS-based received signal at the $p$-th antenna of the BS is given by (8) at the top of the next page.

Let $\mathbf{v} \in\left\{\mathbf{x}_{q}, \mathbf{y}_{p}, \boldsymbol{\omega}_{p}\right\} \in \mathbb{C}^{M N \times 1}$, where $\mathbf{x}_{q}, \mathbf{y}_{p}$, and $\boldsymbol{\omega}_{p}$ denote the vector of transmitted symbols from the $q$-th user, the received signal vector at the $p$-th antenna of the BS, and the corresponding noise vector, respectively. The $(k+M l)$-th element of $\mathbf{v}$ is

$$
\mathbf{v}(k+M l)=v[k, l]
$$

where $v[k, l] \in\left\{x_{q}[k, l], y_{p}[k, l], \omega_{p}[k, l]\right\}$. Then, based on (8), the final received signal from all users to the $p$-th antenna at the $\mathrm{BS}$ is given by

$$
\mathbf{y}_{p}=\sum_{q=1}^{K_{u}} \mathbf{H}_{p, q} \mathbf{x}_{q}+\boldsymbol{\omega}_{p},
$$

where the elements of $\mathbf{x}_{q}$ are i.i.d. with zero mean and variance $P_{q}$. The matrix $\mathbf{H}_{p, q} \in \mathbb{C}^{M N \times M N}$ is a doublyblock circulant matrix, consisting of $N \times N$ sub-blocks each of which is an $M \times M$ circulant matrix, i.e.,

$$
\mathbf{H}_{p, q}=\operatorname{Circ}\left\{\mathbf{H}_{p, q}[0], \ldots, \mathbf{H}_{p, q}[N-1]\right\},
$$

where Circ \{\} denotes the block circulant operator. The subblock circulant matrix $\mathbf{H}_{p, q}[j] \in \mathbb{C}^{M \times M}$, for $j=0, \ldots, N-$ 1 , is defined as (11) at the top of the next page, where $\mathbf{h}_{p, q}(i)$ 


$$
y_{p}[k, l]=\frac{1}{\sqrt{N_{b}}} \sum_{q=1}^{K_{u}} \sum_{i=1}^{M_{q}} h_{q, i} e^{j 2 \pi(p-1) \frac{d_{a} \sin \left(\theta_{q, i}\right)}{\lambda}} e^{-j 2 \pi \frac{k_{q, i}}{M} \frac{l_{q, i}}{N}} x_{q}\left[\left(k-k_{q, i}\right)_{M},\left(l-l_{q, i}\right)_{N}\right]+\omega_{p}[k, l] .
$$

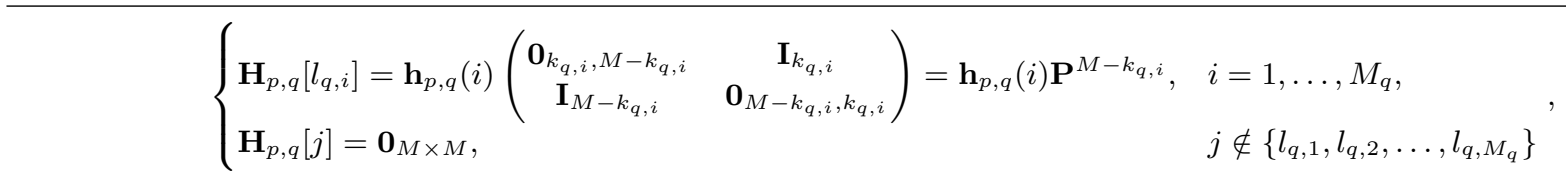

$$
\mathbf{h}_{p, q}=\left(\frac{1}{\sqrt{N_{b}}} h_{q, 1} e^{j 2 \pi(p-1) \frac{d_{a} \sin \left(\theta_{q, 1}\right)}{\lambda}} e^{-j 2 \pi \frac{k_{q, 1}}{M} \frac{l_{q, 1}}{N}}, \ldots, \quad \frac{1}{\sqrt{N_{b}}} h_{q, M_{q}} e^{j 2 \pi(p-1) \frac{d_{a} \sin \left(\theta_{q, M_{q}}\right)}{\lambda}} e^{-j 2 \pi \frac{k_{q, M_{q}}}{M} \frac{l_{q, M_{q}}}{N}}\right)^{T} .
$$

denotes the $i$-th element of the vector $\mathbf{h}_{p, q} \in \mathbb{C}^{M_{q} \times 1}$, which is given by (12). The matrix $\mathbf{P} \in \mathbb{R}^{M \times M}$ is an $M \times M$ circulant permutation matrix, where 1 appears only on the upper diagonal and in the lower left-hand corner, and zeros appear in all other positions; here $\mathbf{P}^{M}=\mathbf{I}_{M}$ [17]. Note that in (11), when $k_{q, i}=l_{q, i}=0$, we have,

$$
\mathbf{H}_{p, q}\left[l_{q, i}\right]=\mathbf{H}_{p, q}[0]=\mathbf{h}_{p, q}(i) \mathbf{I}_{M} .
$$

From (10), we can observe that only $M_{q}$ out of the $N$ circulant matrices $\mathbf{H}_{p, q}[j]$ are non-zero, which clearly shows that the effective channel $\mathbf{H}_{p, q}$ from the $q$-the user to the $p$-th antenna of the BS is extremely sparse.

Let

$$
\begin{aligned}
& \mathbf{y}=\left(\begin{array}{lll}
\mathbf{y}_{1}^{T} & \ldots & \mathbf{y}_{N_{b}}^{T}
\end{array}\right)^{T} \in \mathbb{C}^{N_{b} M N \times 1} \\
& \mathbf{x}=\left(\begin{array}{llll}
\mathbf{x}_{1}^{T} & \mathbf{x}_{2}^{T} & \ldots & \mathbf{x}_{K_{u}}^{T}
\end{array}\right)^{T} \in \mathbb{C}^{K_{u} M N \times 1} \\
& \boldsymbol{\omega}=\left(\begin{array}{lll}
\boldsymbol{\omega}_{1}^{T} & \ldots & \boldsymbol{\omega}_{N_{b}}^{T}
\end{array}\right)^{T} \in \mathbb{C}^{N_{b} M N \times 1},
\end{aligned}
$$

such that the final input-output relation from all users to the $\mathrm{BS}$ is

$$
\mathbf{y}=\mathbf{H x}+\boldsymbol{\omega},
$$

where the block channel matrix $\mathbf{H} \in \mathbb{C}^{N_{b} M N \times K_{u} M N}$ consists of $N_{b} \times K_{u}$ sub-blocks, in which the $(p, q)$-th sub-block is the block matrix $\mathbf{H}_{p, q}$ with the definition given in (10).

In the next section, we will provide some key results which will be used for analyzing the system performance in Section IV.

\section{Key Results}

\section{A. Eigenvalue Decomposition of Channel Matrices}

From [5], the doubly-block circulant matrix $\mathbf{H}_{p, q}$ in (10) can be decomposed as

$$
\mathbf{H}_{p, q}=\left(\mathbf{F}_{N} \otimes \mathbf{F}_{M}\right)^{H} \mathbf{D}_{p, q}\left(\mathbf{F}_{N} \otimes \mathbf{F}_{M}\right)
$$

where $(\cdot)^{H}$ denotes the conjugate transpose. The matrix $\mathbf{F}_{X}=\left(\frac{1}{\sqrt{X}} e^{-j 2 \pi \frac{j k}{X}}\right)_{j, k=0, \ldots X-1}$ denotes the unitary DFT matrix of dimension $X \times X$. The operator $\otimes$ denotes the Kronecker product of two matrices. The diagonal matrix $\mathbf{D}_{p, q}$ can be expressed as

$$
\mathbf{D}_{p, q}=\sum_{i=1}^{M_{q}}\left(\boldsymbol{\Omega}_{N}\right)^{l_{q_{i}}} \otimes \mathbf{D}_{\left[l_{q_{i}}\right]}^{p, q},
$$

where $\boldsymbol{\Omega}_{N}=\operatorname{diag}\left\{1, e^{j 2 \pi \frac{1}{N}}, \ldots, e^{j 2 \pi \frac{N-1}{N}}\right\} \in \mathbb{C}^{N \times N}$ and $\operatorname{diag}\{\}$ denotes the diagonal operator. The diagonal matrix $\mathbf{D}_{\left[l q_{i}\right]}^{p, q} \in \mathbb{C}^{M \times M}$ includes the eigenvalues of the matrix $\mathbf{H}_{p, q}\left[l_{q_{i}}\right]$, and has the form

$\mathbf{D}_{\left[l_{q_{i}}\right]}^{p, q}=\mathbf{h}_{p, q}(i) \operatorname{diag}\left\{\operatorname{eig}\left(\mathbf{P}^{M-k_{q, i}}\right)\right\}, i=1, \ldots, M_{q}$,

where eig $\left(\mathbf{P}^{M-k_{q, i}}\right)$ is the vector containing all eigenvalues of $\mathbf{P}^{M-k_{q, i}}$. It is well-known that the eigenvalues of the permutation matrix $\mathbf{P}$ are precisely the $M$ distinct complex roots of $z^{M}=1$ [17], and, thus, after reordering the eigenvalues, the $m$-th eigenvalue of $\mathbf{P}^{M-k_{q, i}}$ is equal to $e^{-j 2 \pi \frac{k_{q, i}}{M} m}$, for all $m=0, \ldots, M-1$.

Next, we write the diagonal matrix $\mathbf{D}_{p, q}$ as a block diagonal matrix with $N$ diagonal blocks, i.e.,

$$
\mathbf{D}_{p, q}=\operatorname{diag}\left\{\mathbf{D}_{p, q}[0], \ldots, \mathbf{D}_{p, q}[N-1]\right\},
$$

where the diagonal matrix $\mathbf{D}_{p, q}[n] \in \mathbb{C}^{M \times M}$, for $n=$ $0, \ldots, N-1$, is given by

$$
\mathbf{D}_{p, q}[n]=\sum_{i=1}^{M_{q}} e^{j 2 \pi \frac{n l_{q, i}}{N}} \mathbf{D}_{\left[l_{q_{i}}\right.}^{p, q} .
$$

In (19), the $m$-th diagonal element of $\mathbf{D}_{p, q}[n]$ is

$$
\begin{aligned}
\left(\mathbf{D}_{p, q}[n]\right)_{m, m} & =\sum_{i=1}^{M_{q}} e^{j 2 \pi \frac{n l_{q, i}}{N}} e^{-j 2 \pi \frac{k_{q, i}}{M} m} \mathbf{h}_{p q}(i) \\
& =\mathbf{e}_{q,(n, m)}^{H} \mathbf{h}_{p, q}, \quad m=0, \ldots, M-1,
\end{aligned}
$$

where the vector $\mathbf{h}_{p, q}$ is given in (12) and the vector $\mathbf{e}_{q,(n, m)} \in \mathbb{C}^{M_{q} \times 1}$ is defined as (21) at the top of the next page.

\section{B. The Multi-path DoA Spread in Massive MIMO}

As we will show in Section IV, the behavior of the term $a_{i, j}^{q, q^{\prime}}=\left(\mathbf{a}\left(\theta_{q, i}\right)\right)^{H} \mathbf{a}\left(\theta_{q^{\prime}, j}\right)$, where the expression for the steering vector $\mathbf{a}\left(\theta_{q, i}\right)$ is given in (6), has a significant impact on the OTFS system performance. Thus, the behavior of the term $a_{i, j}^{q, q^{\prime}}$ will be studied under a massive number of BS antennas in this section.

Firstly, recalling (6), we have

$$
\begin{aligned}
a_{i, j}^{q, q^{\prime}} & =\frac{1}{N_{b}} \sum_{p=1}^{N_{b}} e^{j 2 \pi(p-1)\left(\frac{d_{a} \sin \left(\theta_{q^{\prime}, j}\right)}{\lambda}-\frac{d_{a} \sin \left(\theta_{q, i}\right)}{\lambda}\right)} \\
& =\frac{1}{N_{b}} \frac{\sin \left(\pi N_{b}\left(\phi_{q^{\prime}, j}-\phi_{q, i}\right)\right)}{\sin \left(\pi\left(\phi_{q^{\prime}, j}-\phi_{q, i}\right)\right)} e^{j \pi\left(N_{b}-1\right)\left(\phi_{q^{\prime}, j}-\phi_{q, i}\right)},
\end{aligned}
$$

where $\phi_{q, i}=\frac{d_{a}}{\lambda} \sin \left(\theta_{q, i}\right)$, for $i=1, \ldots, M_{q}$ and $q=$ $1, \ldots, K_{u}$. In the following, we consider the special case where the spacing between any two adjacent antennas of the BS is $d_{a}=\frac{\lambda}{2}$, i.e., $\phi_{q, i} \in\left[-\frac{1}{2}, \frac{1}{2}\right)$. In this case, it is always possible to write

$$
\phi_{q, i}=\frac{1}{2} \sin \left(\theta_{q, i}\right)=-\frac{1}{2}+\frac{n_{q, i}}{N_{b}}+\frac{\Delta_{q, i}}{N_{b}},
$$

where $n_{q, i} \in\left\{0,1, \ldots, N_{b}-1\right\}$, and the notation $\Delta_{q, i} \in$ $(0,1)$ denotes the off-grid gap. Note that the special case $\Delta_{q, i}=0$, which represents the DFT-based sample method and produces orthogonal steering vectors, represents an ideal case. Substituting (23) into (22), the magnitude in (22) has 


$$
\mathbf{e}_{q,(n, m)}=\left(e^{-j 2 \pi \frac{n l_{q, 1}}{N}} e^{j 2 \pi \frac{m k_{q, 1}}{M}}, \quad \ldots, \quad e^{-j 2 \pi \frac{n l_{q, M_{q}}}{N}} e^{j 2 \pi \frac{m k_{q, M_{q}}}{M}}\right)^{T}
$$

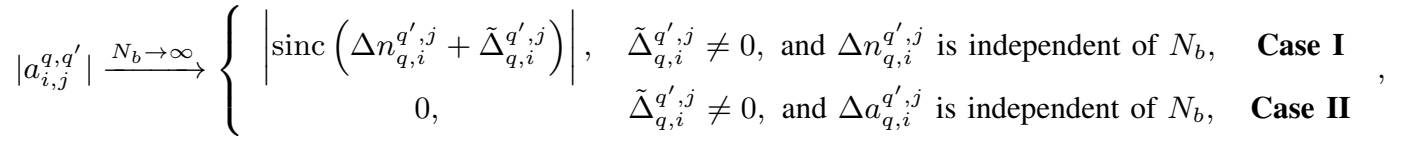

the property

$$
\left|a_{i, j}^{q, q^{\prime}}\right|=\left\{\begin{array}{ll}
1, & n_{q^{\prime}, j}=n_{q, i}, \text { and } \Delta_{q^{\prime}, j}=\Delta_{q, i} \\
0, & n_{q^{\prime}, j} \neq n_{q, i}, \text { and } \Delta_{q^{\prime}, j}=\Delta_{q, i}
\end{array},\right.
$$

which also corresponds to an ideal case as orthogonal steering vectors are produced.

However, under a more general case, the asymptotic result in (25) at the top of this page, can be obtained under a massive number of BS antennas, where $\operatorname{sinc}(x)=\frac{\sin (\pi x)}{\pi x}$ is the sinc function. The parameters $\tilde{\Delta}_{q, i}^{q^{\prime}, j}, \Delta n_{q, i}^{q^{\prime}, j}$ and $\Delta a_{q, i}^{q^{\prime}, j}$ are defined as

$$
\begin{aligned}
& \tilde{\Delta}_{q, i}^{q^{\prime}, j} \triangleq \Delta_{q^{\prime}, j}-\Delta_{q, i} \in(-1,1), \\
& \Delta n_{q, i}^{q^{\prime, j}} \triangleq n_{q^{\prime}, j}-n_{q, i} \in \mathbb{Z} \in\left[-N_{b}+1, N_{b}-1\right], \\
& \Delta a_{q, i}^{q^{\prime}, j} \triangleq \frac{1}{N_{b}}\left(n_{q^{\prime}, j}-n_{q, i}\right) \in\left(-1+\frac{1}{N_{b}}, 0\right) \cup\left(0,1-\frac{1}{N_{b}}\right) .
\end{aligned}
$$

From (23), the BS can create $N_{b}$ orthogonal beams. The parameter $\Delta n_{q, i}^{q^{\prime}, j}$ of (25) represents the interger distance between any two given paths, i.e., the difference of the beams that the two paths occupy. Observing Fig. $1, \Delta n_{q, i}^{q^{\prime}, j}=0$ corresponds to the main lobe in Fig. $1, \Delta n_{q, i}^{q^{\prime}, j}= \pm 1$ correspond to the first two sidelobes in Fig. 1, etc. It can be observed from Fig. 1 that the peaks of Case I in (25) decrease with respect to $\left|\Delta n_{q, i}^{q^{\prime}, j}\right|$. Thus, we can set a threshold on the peaks' value $0<\rho_{\text {thres }}<1$, which is used to determine the main dominant lobes that highly affect the performance, i.e., the region of $\Delta n_{q, i}^{q^{\prime}, j}$. Then, by solving the following inequality

$$
\left|\operatorname{sinc}\left(n_{\text {thres }}+\frac{1}{2}\right)\right| \geq \rho_{\text {thres }}
$$

an absolute threshold $n_{\text {thres }}$ can be obtained. Combining Fig. 1 , we only identify the path-pairs that fall within the lobes $\Delta n_{q, i}^{q^{\prime}, j} \in\left[-n_{\text {thres }}+1, n_{\text {thres }}-1\right]$. After determining the main lobes, the parameter $\tilde{\Delta}_{q, i}^{q^{\prime}, j}$ of (25) is used to find the exact correlation for any two given paths, i.e., corresponding to the green shaded region in Fig. 1.

Assume that the values $n_{q, i}$ are independent for different $(q, i)$, and are uniformly distributed in the set $\left\{0,1, \ldots, N_{b}-\right.$ $1\}$. Then, the probability that any two given paths that fall within the region $\left[-n_{\text {thres }}+1, n_{\text {thres }}-1\right]$ is

$$
\begin{aligned}
& \operatorname{Pr}\left\{-n_{\text {thres }}+1 \leq \Delta n_{q, i}^{q^{\prime}, j} \leq n_{\text {thres }}-1\right\} \\
= & \frac{\left[N_{b}-2\left(n_{\text {thres }}-1\right)-2\right]\left[2\left(n_{\text {thres }}-1\right)+1\right]+3 n_{\text {thres }}^{2}-n_{\text {thres }}}{N_{b}^{2}} \\
= & \frac{\left(2 N_{b}-n_{\text {thres }}\right)\left(n_{\text {thres }}-1\right)+N_{b}}{N_{b}^{2}}
\end{aligned}
$$

which obviously approaches zero as $N_{b} \rightarrow \infty$. Thus, with massive MIMO, the probability of any two given paths being strongly correlated is low (lower than (30) due to the fact that this strong correlation corresponds to the green shaded region in Fig. 1). Therefore, in the following, the

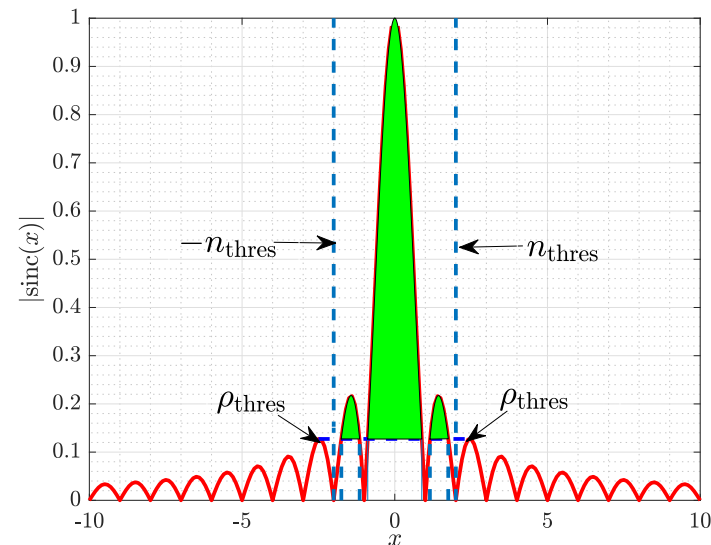

Fig. 1: The value of $|\operatorname{sinc}(x)|$ versus $x$ with $N_{b}=10$, where $x=\Delta n_{q, i}^{q^{\prime}, j}+\tilde{\Delta}_{q, i}^{q^{\prime}, j}$.

performance analysis will be presented for this low but nonzero probability scenario.

\section{LOW COMPLEXITY APPROXIMATION FOR ZERO-FORCING (ZF) DECODING SCHEME}

When the ZF scheme is applied at the BS, the corresponding decoding matrix $\mathbf{R}^{\mathrm{zf}} \in \mathbb{C}^{K_{u} M N \times N_{b} M N}$ is given by

$$
\mathbf{R}^{\mathrm{zf}}=\left(\mathbf{H}^{H} \mathbf{H}\right)^{-1} \mathbf{H}^{H} \text {. }
$$

Pre-multiplying both sides of (14) with $\mathbf{R}^{\mathrm{zf}}$, the received signal at the BS after using $\mathrm{ZF}$ is

$$
\tilde{\mathbf{y}}^{\mathrm{zf}}=\mathbf{R}^{\mathrm{zf}} \mathbf{y}=\mathbf{x}+\left(\mathbf{H}^{H} \mathbf{H}\right)^{-1} \mathbf{H}^{H} \boldsymbol{\omega} .
$$

From (32), the received sample corresponding to the symbol $x_{q}[k, l]$ of the $q$-th user is

$$
\begin{aligned}
& \tilde{\mathbf{y}}^{\mathrm{zf}}(k+M((q-1) N+l)) \\
= & \mathbf{x}_{q}(k, l)+\left(\left(\left(\left(\mathbf{H}^{H} \mathbf{H}\right)^{-1} \mathbf{H}^{H}\right)_{[q],:}\right)_{[l],:}\right)_{k,:} \boldsymbol{\omega},
\end{aligned}
$$

where $(\mathbf{A})_{[i],:}$ and $(\mathbf{B})_{i,:}$ denote the $i$-th block row of the block matrix $\mathbf{A}$ and the $i$-th row of the matrix $\mathbf{B}$, respectively. Using (33), the signal-to-noise-ratio (SNR) for the $q$-th user is given by

$$
\mathrm{SNR}_{q}^{\mathrm{zf}}=\frac{P_{q}}{\sigma^{2}} \frac{1}{\left(\left(\left(\left(\mathbf{H}^{H} \mathbf{H}\right)^{-1}\right)_{[q],[q]}\right)_{[l],[l]}\right)_{k, k}}
$$

where $(\mathbf{A})_{[i],[j]}$ and $(\mathbf{B})_{i, j}$ denote the $(i, j)$-th sub-block of the block matrix $\mathbf{A}$ and the $(i, j)$-th element of the matrix B, respectively.

From (34), we need to calculate the term $\left(\mathbf{H}^{H} \mathbf{H}\right)^{-1}$. First, we rewrite the channel vector $\mathbf{h}_{p, q}$ of (12) as

$$
\mathbf{h}_{p, q}=\mathbf{V}_{q} \boldsymbol{\Psi}_{p, q} \boldsymbol{\Xi}_{q} \tilde{\mathbf{h}}_{q}
$$

where $\mathbf{V}_{q} \in \mathbb{C}^{M_{q} \times M_{q}}, \boldsymbol{\Psi}_{p, q} \in \mathbb{C}^{M_{q} \times M_{q}}$, and $\boldsymbol{\Xi}_{q} \in$ $\mathbb{C}^{M_{q} \times M_{q}}$ are all diagonal matrices, in which the $i$-th diagonal elements are $\left(\mathbf{V}_{q}\right)_{i, i}=e^{-j 2 \pi \frac{k_{q, i}}{M} \frac{l_{q, i}}{N}},\left(\mathbf{\Psi}_{p, q}\right)_{i, i}=$ $\frac{1}{\sqrt{N_{b}}} e^{j 2 \pi(p-1) \frac{d_{a} \sin \left(\theta_{q, i}\right)}{\lambda}}$, and $\left(\boldsymbol{\Xi}_{q}\right)_{i, i}=\beta_{q, i}$, respectively. 


$$
\mathbf{D}_{[q],\left[q^{\prime}\right]}=\sum_{p=1}^{N_{b}} \mathbf{D}_{p, q}^{H} \mathbf{D}_{p, q^{\prime}}=\operatorname{diag}\left\{\sum_{p=1}^{N_{b}}\left(\mathbf{D}_{p, q}[0]\right)^{H}\left(\mathbf{D}_{p, q^{\prime}}[0]\right), \ldots, \sum_{p=1}^{N_{b}}\left(\mathbf{D}_{p, q}[N-1]\right)^{H}\left(\mathbf{D}_{p, q^{\prime}}[N-1]\right)\right\} \in \mathbb{C}^{M N \times M N} .
$$

$$
\left(\tilde{\mathbf{A}}_{(n, m)}^{q, q^{\prime}}\right)_{i, j}=e^{j 2 \pi n\left(\frac{l_{q^{\prime}, j}}{N}-\frac{l_{q, i}}{N}\right)} e^{j 2 \pi m\left(\frac{k_{q, i}}{M}-\frac{k_{q^{\prime}, j}}{M}\right)} e^{j 2 \pi\left(\frac{k_{q, i}}{M} \frac{l_{q, i}}{N}-\frac{k_{q^{\prime}, j} l^{l} q^{\prime}, j}{N}\right)}\left(\left(\mathbf{a}\left(\theta_{q, i}\right)\right)^{H} \mathbf{a}\left(\theta_{q^{\prime}, j}\right)\right) .
$$

The random vector $\tilde{\mathbf{h}}_{q}=\left(\begin{array}{lll}\tilde{h}_{q, 1}, \ldots, & \tilde{h}_{q, M_{q}}\end{array}\right)^{T} \in \mathbb{C}^{M_{q} \times 1}$ is distributed as $\tilde{\mathbf{h}}_{q} \sim \mathcal{C N}\left(\mathbf{0}, \mathbf{I}_{M_{q}}\right)$.

The $\left(q, q^{\prime}\right)$-th block of the Wishart block matrix $\mathbf{H}^{H} \mathbf{H}$ is $\sum_{p=1}^{N_{b}} \mathbf{H}_{p, q}^{H} \mathbf{H}_{p, q^{\prime}} \in \mathbb{C}^{M N \times M N}$, in which $q, q^{\prime} \in\left\{1, \ldots, K_{u}\right\}$ With the eigenvalue decomposition of $\mathbf{H}_{p, q}$ in (15), we have $\mathbf{H}^{H} \mathbf{H}=\left[\mathbf{I}_{K_{u}} \otimes\left(\mathbf{F}_{N} \otimes \mathbf{F}_{M}\right)^{H}\right] \mathbf{D}\left[\mathbf{I}_{K_{u}} \otimes\left(\mathbf{F}_{N} \otimes \mathbf{F}_{M}\right)\right]$, (36) where $\mathbf{D}$ is a $K_{u} \times K_{u}$ block matrix with the $\left(q, q^{\prime}\right)$-th subblock given in (37) at the top of this page. With (19) and (20), the $m$-th diagonal element of the $n$-th diagonal sub-block of (37), for $n=0, \ldots, N-1, m=0, \ldots, M-1$, is given by

$$
\begin{aligned}
& \left(\sum_{p=1}^{N_{b}}\left(\mathbf{D}_{p, q}[n]\right)^{H}\left(\mathbf{D}_{p, q^{\prime}}[n]\right)\right)_{m, m} \\
= & \sum_{p=1}^{N_{b}} \mathbf{h}_{p, q}^{H} \mathbf{e}_{q,(n, m)} \mathbf{e}_{q^{\prime},(n, m)}^{H} \mathbf{h}_{p, q^{\prime}}=\tilde{\mathbf{h}}_{q}^{H} \mathbf{\Xi}_{q}^{H} \tilde{\mathbf{A}}_{(n, m)}^{q, q^{\prime}} \mathbf{\Xi}_{q^{\prime}} \tilde{\mathbf{h}}_{q^{\prime}},
\end{aligned}
$$

where the matrix $\tilde{\mathbf{A}}_{(n, m)}^{q, q^{\prime}} \in \mathbb{C}^{M_{q} \times M_{q^{\prime}}}$ is defined as

$$
\tilde{\mathbf{A}}_{(n, m)}^{q, q^{\prime}} \triangleq \sum_{p=1}^{N_{b}} \boldsymbol{\Psi}_{p, q}^{H} \mathbf{V}_{q}^{H} \mathbf{e}_{q,(n, m)} \mathbf{e}_{q^{\prime},(n, m)}^{H} \mathbf{V}_{q^{\prime}} \boldsymbol{\Psi}_{p, q^{\prime}} .
$$

From (36), in order to calculate $\left(\mathbf{H}^{H} \mathbf{H}\right)^{-1}$, we only need to calculate the term $\mathbf{D}^{-1}$, which is always possible as $\mathbf{D}$ is invertible [18]. Observing (39), the $(i, j)$-th element of $\tilde{\mathbf{A}}_{(n, m)}^{q, q^{\prime}}$ is given by (40) at the top of this page. Note that the behavior of the term $\left(\mathbf{a}\left(\theta_{q, i}\right)\right)^{H} \mathbf{a}\left(\theta_{q^{\prime}, j}\right)$ has been analyzed in Section III-B. Due to the effect of Case I in (25), the block matrix D may not always be diagonalizable, even in the case of a massive number of antennas. Therefore, a low-complexity approximation of $\mathbf{D}^{-1}$ is provided in the following.

\section{A. Low Complexity Approximation of $\mathbf{D}^{-1}$}

From [19], for a given precondition matrix $\mathbf{B}$, if

$$
\lim _{n \rightarrow \infty}\left(\mathbf{I}-\mathbf{B}^{-1} \mathbf{D}\right)^{n}=\mathbf{0},
$$

or equivalently, if the spectral radius of $\mathbf{I}-\mathbf{B}^{-1} \mathbf{D}$ satisfies

$$
\rho\left(\mathbf{I}-\mathbf{B}^{-1} \mathbf{D}\right)<1,
$$

the inverse of $\mathbf{D}$ can be expressed by a Neumann series, which is given by

$$
\mathbf{D}^{-1}=\left(\mathbf{B}^{-1} \mathbf{D}\right)^{-1} \mathbf{B}^{-1}=\sum_{n=0}^{\infty}\left(\mathbf{I}-\mathbf{B}^{-1} \mathbf{D}\right)^{n} \mathbf{B}^{-1}
$$

Truncating this summation, an approximation of $\mathbf{D}^{-1}$ is given by

$$
\mathbf{D}^{-1} \approx \tilde{\mathbf{D}}_{L}^{-1} \triangleq \sum_{n=0}^{L-1}\left(\mathbf{I}-\mathbf{B}^{-1} \mathbf{D}\right)^{n} \mathbf{B}^{-1},
$$

which will be more accurate (and require higher computational complexity) for larger $L$.

Thus, the choice of the precondition matrix $\mathbf{B}$ is critical for the performance analysis. In this paper, the block matrix
B is designed as

$$
\mathbf{B}=\mathbf{B}_{\mathrm{d}}+\omega \mathbf{B}_{\mathrm{c}},
$$

where the coefficient $0 \leq \omega \leq 1$. When the block matrix $\mathbf{D}$ is diagonally dominant, then $\omega$ needs to be chosen to be close to zero in order to satisfy (42). The matrix $\mathbf{B}_{\mathrm{d}}=\operatorname{diag}(\mathbf{D})$, i.e., the $i$-th diagonal sub-block is

$$
\left(\mathbf{B}_{\mathrm{d}}\right)_{[i],[i]}=(\mathbf{D})_{[i],[i]}=\sum_{p=1}^{N_{b}} \mathbf{D}_{p, i}^{H} \mathbf{D}_{p, i} .
$$

The block matrix $\mathbf{B}_{\mathrm{c}}$ is defined as

$$
\left(\mathbf{B}_{\mathrm{c}}\right)_{[i],[j]}=\left\{\begin{array}{cc}
(\mathbf{D})_{[i],[j]}, & i \neq q^{\max }, j=q^{\max } \\
\mathbf{0} & \text { otherwise }
\end{array},\right.
$$

where the index $q^{\max }$ is obtained from

$$
\arg \max _{1 \leq j \leq K_{u}} \sum_{\substack{i=1 \\ i \neq j}}^{K_{u}} \sum_{m=1}^{M N}\left|\left((\mathbf{D})_{[i],[j]}\right)_{m, m}\right|,
$$

which indicates that the $q^{\max }$-th user, which has the largest cumulative correlations with the residual $K_{u}-1$ users, is selected.

In the following, to simplify the performance analysis and reduce the complexity, we take $L=2$ in (44). Then, ${ }^{1}$

$$
\mathbf{D}^{-1} \approx \tilde{\mathbf{D}}_{2}^{-1}=2 \mathbf{B}^{-1}-\mathbf{B}^{-1} \mathbf{D} \mathbf{B}^{-1},
$$

where

$$
\mathbf{B}^{-1}=\left(\mathbf{B}_{\mathrm{d}}+\omega \mathbf{B}_{\mathrm{c}}\right)^{-1}=\left(\mathbf{I}-\omega \mathbf{B}_{\mathrm{d}}^{-1} \mathbf{B}_{\mathrm{c}}\right) \mathbf{B}_{\mathrm{d}}^{-1} .
$$

It is known that the computational complexity of calculating $\mathbf{D}^{-1}$ by using Gaussian elimination is with the order $\mathcal{O}\left(K_{u}^{3} M^{3} N^{3}\right)$. However, the approximation result in (49) is obtained with a complexity of the order $\mathcal{O}\left(K_{u}^{2} M N\right)$, which is significantly lower than $\mathcal{O}\left(K_{u}^{3} M^{3} N^{3}\right)$, especially for very large $M$ and $N$.

\section{B. Performance Analysis}

Theorem 1: Under the ZF scheme and with the Neumann series approximation $(L=2$ in (44)), the SNR in (34) for decoding the signal of the $q$-th user can be approximated as

$$
\mathrm{SNR}_{q}^{\mathrm{zf}} \approx{\widetilde{\mathrm{SNR}_{q}^{\mathrm{zf}}}}_{q}^{\mathrm{z}}=\frac{P_{q}}{\sigma^{2}} \frac{1}{g_{q}^{\mathrm{zf}}},
$$

where $g_{q}^{\mathrm{zf}}$ is

$$
g_{q}^{\mathrm{zf}}= \begin{cases}(53), & q=q^{\max } \\ (54), & q \neq q^{\max }\end{cases}
$$

in which $q^{\max }$ is determined by (48). The results (53) and (54) are given at the top of the next page.

Proof: See Appendix A.

From Theorem 1, we can see that the performance is highly dependent on the matrix $\tilde{\mathbf{A}}_{(n, m)}^{q, q^{\prime}}$, for $q, q^{\prime}=1, \ldots, K_{u}, n=$ $0, \ldots, N-1$ and $m=0, \ldots, M-1$, which we call the OTFS-MPCM. From (39) and (40), the multi-path delay $k_{q, i}$ and Doppler shift $l_{q, i}$, and the total number of blocks in the

\footnotetext{
${ }^{1}$ In order to use the approximation in (49), we need to prove the convergence condition in (42), which is omitted in this paper due to the space limitation. It can be shown that this convergence condition holds with high probability in the massive MIMO regime.
} 
$g_{q}^{z \mathbf{f}}=\frac{1}{N} \frac{1}{M} \sum_{n=0}^{N-1} \sum_{m=0}^{M-1}\left\{\left(\tilde{\mathbf{h}}_{q}^{H} \mathbf{\Xi}_{q}^{H} \tilde{\mathbf{A}}_{(n, m)}^{q, q} \mathbf{\Xi}_{q} \tilde{\mathbf{h}}_{q}\right)^{-1}+\omega\left(\tilde{\mathbf{h}}_{q}^{H} \mathbf{\Xi}_{q}^{H} \tilde{\mathbf{A}}_{(n, m)}^{q, q} \boldsymbol{\Xi}_{q} \tilde{\mathbf{h}}_{q}\right)^{-2} \sum_{\substack{q^{\prime}=1 \\ q^{\prime} \neq q}}^{K_{u}}\left[\left|\tilde{\mathbf{h}}_{q}^{H} \mathbf{\Xi}_{q}^{H} \tilde{\mathbf{A}}_{(n, m)}^{q, q^{\prime}} \mathbf{\Xi}_{q^{\prime}} \tilde{\mathbf{h}}_{q^{\prime}}\right|^{2}\left(\tilde{\mathbf{h}}_{q^{\prime}}^{H} \mathbf{\Xi}_{q^{\prime}}^{H} \tilde{\mathbf{A}}_{(n, m)}^{q^{\prime}, q^{\prime}} \mathbf{\Xi}_{q^{\prime}} \tilde{\mathbf{h}}_{q^{\prime}}\right)^{-1}\right]\right\}$

$g_{q}^{2 f}=\frac{1}{N} \frac{1}{M} \sum_{n=0}^{N-1} \sum_{m=0}^{M-1}\left\{\left(\tilde{\mathbf{h}}_{q}^{H} \boldsymbol{\Xi}_{q}^{H} \tilde{\mathbf{A}}_{(n, m)}^{q, q} \boldsymbol{\Xi}_{q} \tilde{\mathbf{h}}_{q}\right)^{-1}+\omega\left(\tilde{\mathbf{h}}_{q}^{H} \boldsymbol{\Xi}_{q}^{H} \tilde{\mathbf{A}}_{(n, m)}^{q, q} \boldsymbol{\Xi}_{q} \tilde{\mathbf{h}}_{q}\right)^{-2}\left|\tilde{\mathbf{h}}_{q}^{H} \boldsymbol{\Xi}_{q}^{H} \tilde{\mathbf{A}}_{(n, m)}^{q, m^{\max }} \boldsymbol{\Xi}_{q^{\max }} \tilde{\mathbf{h}}_{q^{\max }}\right|^{2}\left(\tilde{\mathbf{h}}_{q^{\max }}^{H} \boldsymbol{\Xi}_{q^{\max }}^{H} \tilde{\mathbf{A}}_{(n, m)}^{q^{\max }, q^{\max }} \boldsymbol{\Xi}_{q^{\max }} \tilde{\mathbf{h}}_{q^{\max }}\right)^{-1}\right\}$

delay and Doppler domain $M$ and $N$, affect only the phase of the entries of the OTFS-MPCM, and these parameters rotate the $(i, j)$-th element of OTFS-MPCM by the phase angle $\varsigma_{i j}$ given by

$$
\begin{gathered}
\varsigma_{i j}=2 \pi\left[n\left(\frac{l_{q^{\prime}, j}}{N}-\frac{l_{q, i}}{N}\right)+m\left(\frac{k_{q, i}}{M}-\frac{k_{q^{\prime}, j}}{M}\right)\right. \\
\left.+\left(\frac{k_{q, i}}{M} \frac{l_{q, i}}{N}-\frac{k_{q^{\prime}, j}}{M} \frac{l_{q^{\prime}, j}}{N}\right)\right] .
\end{gathered}
$$

However, from (22), the direction of arrival $\theta_{q, i}$ not only affects its magnitude but also rotates its phase; the magnitude of the $(i, j)$-th element of OTFS-MPCM is $\left|a_{i, j}^{q, q^{q}}\right|$, while the corresponding phase rotation is given by $\pi\left(N_{b}-\right.$ 1) $\left(\phi_{q^{\prime}, j}-\phi_{q, i}\right)$. In addition, the OTFS-MPCM $\tilde{\mathbf{A}}_{(n, m)}^{q, q^{\prime}}$, for $q \neq q^{\prime}$, will become more sparse as the number of BS antennas increases. The DoA effect can be clearly shown via an example where $M_{q}=1$ for all $q=1, \ldots, K_{u}$ as follows.

Example 1: $q=q^{\max }$ and $\left\{M_{q^{\prime}}=1\right\}_{q^{\prime}=1}^{K_{u}}$ : This means that each user transmits its signal only via the direct link. In this case, the delay and Doppler shift are set as $k_{q, 1}=l_{q, 1}=0$. Then, we have

$$
\begin{aligned}
& \widehat{\operatorname{SNR}}_{q}^{\mathrm{zf}}=\operatorname{SNR}_{q}^{\mathrm{zf}, \text { one }} \\
& =\frac{P_{q} \beta_{q, 1}^{2}\left|\tilde{\mathbf{h}}_{q}(1)\right|^{2}}{\sigma^{2}}\left[1+\omega\left(\sum_{\substack{q^{\prime}=1 \\
q^{\prime} \neq q}}^{K_{u}}\left|\operatorname{sinc}\left(\Delta n_{q, 1}^{q^{\prime}, 1}+\tilde{\Delta}_{q, 1}^{q^{\prime}, 1}\right)\right|^{2}\right)\right]^{-1}
\end{aligned}
$$

This quantitatively shows the negative effect of multi-user correlations, which are strongly dependent on the locations of the users and BS.

\section{Simulation Results}

Our simulations are conducted under the following parameters setting unless otherwise stated. We define the parameter $\mathrm{SNR}=\frac{P_{q}}{\sigma^{2}}=10 \mathrm{~dB}$ and the number of paths for each user is $M_{q}=5$, for all $q=1, \ldots, K_{u}$. The total number of blocks in the delay and Doppler domain are $M=8$ and $N=8$, respectively. The carrier frequency and sub-carrier spacing are set as $f_{c}=4 \mathrm{GHz}$ and $\Delta f=1 / T=15 \mathrm{KHz}$, respectively. Other parameters are given in Table I.

TABLE I: Numerical Setting

\begin{tabular}{|c|c|c|c|c|c|}
\hline User number & \multicolumn{5}{|c|}{1} \\
\hline Channel path number & 1 & 2 & 3 & 4 & 5 \\
\hline Delay $\tau_{q, i}(\mu s)$ & 1.01 & 14.05 & 24.07 & 30.04 & 34.06 \\
\hline Doppler shift $\nu_{q, i}(\mathrm{~Hz})$ & 1500 & 1900 & 3760 & 5635 & 7600 \\
\hline User number & \multicolumn{5}{|c|}{2} \\
\hline Channel path number & 1 & 2 & 3 & 4 & 5 \\
\hline Delay $\tau_{q, i}(\mu s)$ & 5.05 & 12.07 & 17.06 & 26.03 & 35.01 \\
\hline Doppler shift $\nu_{q, i}(\mathrm{~Hz})$ & 1421 & 1938 & 4258 & 6012 & 7531 \\
\hline User number & \multicolumn{5}{|c|}{3} \\
\hline Channel path number & 1 & 2 & 3 & 4 & 5 \\
\hline Delay $\tau_{q, i}(\mu s)$ & 8.04 & 15.05 & 23.08 & 28.45 & 33.78 \\
\hline Doppler shift $\nu_{q, i}(\mathrm{~Hz})$ & 783 & 2024 & 3948 & 5839 & 7556 \\
\hline
\end{tabular}
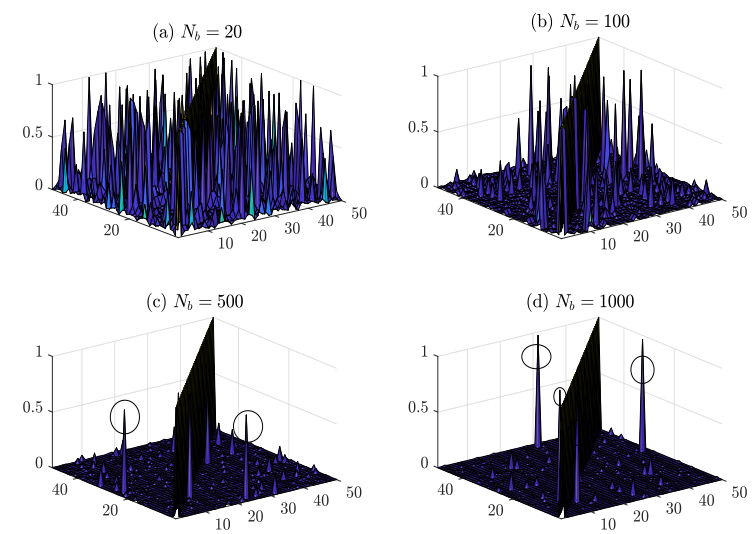

Fig. 2: The $K_{u} M_{q} \times K_{u} M_{q}$ multi-path correlations with $K_{u}=10$.

\section{A. The DoA Effect in Massive MIMO}

To show the effect of the direction of arrival (DoA) $\theta_{q, i}$ clearly, we stack the steering vectors $\mathbf{a}\left(\theta_{q, i}\right)$ of (6) from all paths of all users to form

$\mathbf{A}=\left(\mathbf{a}\left(\theta_{1,1}\right), \ldots, \mathbf{a}\left(\theta_{1, M_{1}}\right), \ldots, \mathbf{a}\left(\theta_{K_{u}, 1}\right), \ldots, \mathbf{a}\left(\theta_{K_{u}, M_{K_{u}}}\right)\right)^{T}$

and define the correlation matrix $\mathbf{Q} \in \mathbb{R}^{K_{u} M_{q} \times K_{u} M_{q}}$ with the $\left(M_{q}(q-1)+i, M_{q^{\prime}}\left(q^{\prime}-1\right)+j\right)$-th element given by

$$
(\mathbf{Q})_{i, j}^{q, q^{\prime}}=\left|\left(\mathbf{A A}^{H}\right)_{i, j}^{q, q^{\prime}}\right|=\left|a_{i, j}^{q, q^{\prime}}\right|
$$

where $a_{i, j}^{q, q^{\prime}}$ is given in (22).

Fig. 2 shows the instantaneous value of each element of the matrix $\mathbf{Q}$. It can be seen that by increasing the number of the BS antennas, most off-diagonal elements approach zero, with the exception of a very small number of elements which still dominate the correlation matrix (identified by circles). This confirms the results in Section III-B, which demonstrated that with massive MIMO at the BS, the value of $\left|a_{i, j}^{q, q^{\prime}}\right|$, for $q \neq q^{\prime}$ or $i \neq j$, is large with a low but nonzero probability. This also shows the low probability case that massive MIMO fails in obtaining FP.

\section{B. Low Complexity ZF Approximation}

Fig. 3 is used to verify the correctness of our approximation obtained by using the Neumann series. In this figure, the spectral efficiency (bits/s/Hz) for the $q$-th user is simulated, which is defined as

$$
C=\log _{2}\left(1+\mathrm{SNR}_{q}^{\mathrm{zf}}\right)
$$

where the exact $\mathrm{SNR}_{q}^{\mathrm{zf}}$ and its approximation $\widetilde{\mathrm{SNR}}_{q}^{\mathrm{zf}}$ are given in (34) and (51), respectively. Meanwhile, $\omega=0$ represents the ideal case that the FP is obtained with massive MIMO. This figure shows that upon increasing the number of antennas at the BS, with high probability most simulation 


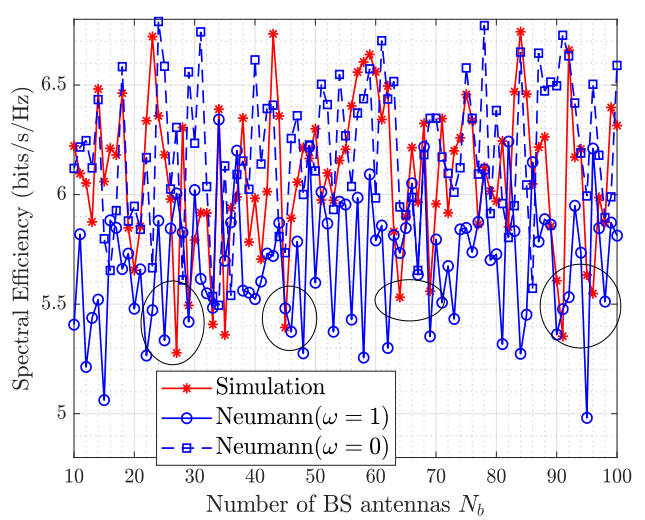

Fig. 3: The simulation and approximation of the spectral efficiency versus the number of BS antennas $N_{b}$ with $K_{u}=3$ for the case $q=q^{\max }$.

results will approach the approximation-based result with $\omega=0$, since massive MIMO can acquire orthogonal channels with high probability. However, there are still some cases (identified by circles) of simulation results that fall near the case of $\omega=1$ or within the region between $\omega=0$ and $\omega=1$, which corroborates the finding that there is a low but non-negligible probability that paths will be correlated for this setting (i.e., FP will not be obtained).

\section{CONCLUSIONS}

This paper has investigated an OTFS-based uplink massive MIMO system, in which multiple users transmit information symbols to the BS in the delay-Doppler domain. The ZF decoding and a ULA were employed at the BS. Different from the existing works, which mainly focused on channel estimation and low-complexity precoder/detector design, a detailed performance analysis has been provided in this paper. In contrast to the existing massive MIMO works, the low but non-zero probability case that massive MIMO fails in offering FP was considered. Due to this reason, the uplink channels from all users cannot be diagonalized with massive MIMO. Thus, the NS expansion based low-complexity approximation of the ZF scheme, was provided. Finally, using the properties of circulant matrices, the OTFS-based multi-path correlation matrix was derived.

\section{APPENDIX A \\ PROOF OF THEOREM 1}

Here we only prove the result for the case $q=q^{\max }$, and a similar process can be used for the other case $q \neq q^{\max }$.

From (36), the $(q, q)$-th sub-block of $\left(\mathbf{H}^{H} \mathbf{H}\right)^{-1}$ is

$$
\left(\left(\mathbf{H}^{H} \mathbf{H}\right)^{-1}\right)_{[q],[q]}=\left(\mathbf{F}_{N} \otimes \mathbf{F}_{M}\right)^{H}\left(\mathbf{D}^{-1}\right)_{[q],[q]}\left(\mathbf{F}_{N} \otimes \mathbf{F}_{M}\right),
$$

where $\left(\mathbf{D}^{-1}\right)_{[q],[q]}$ can be approximated by using (49), yielding

$$
\begin{aligned}
& \left(\mathbf{D}^{-1}\right)_{[q],[q]} \approx\left(\tilde{\mathbf{D}}_{2}^{-1}\right)_{[q],[q]}=\left(\mathbf{D}_{[q],[q]}\right)^{-1} \\
& +\omega\left(\mathbf{D}_{[q],[q]}\right)^{-1} \sum_{\substack{q^{\prime}=1 \\
q^{\prime} \neq q}}^{K_{u}}\left\{\mathbf{D}_{[q],\left[q^{\prime}\right]}\left(\mathbf{D}_{\left[q^{\prime}\right],\left[q^{\prime}\right]}\right)^{-1} \mathbf{D}_{[q],\left[q^{\prime}\right]}^{H}\right\}\left(\mathbf{D}_{[q],[q]}\right)^{-1} .
\end{aligned}
$$

Using (37) and (38), it is easy to obtain that the approximation in (60) is also diagonal. Thus, from (59), we have

$$
\begin{aligned}
& \left(\left(\left(\left(\mathbf{H}^{H} \mathbf{H}\right)^{-1}\right)_{[q],[q]}\right)_{[l],[l]}\right)_{k, k} \\
= & \frac{1}{N} \frac{1}{M} \sum_{n, n^{\prime}=0}^{N-1} \sum_{m, m^{\prime}=0}^{M-1}\left[\times\left(\left(\left(\mathbf{D}^{-1}\right)_{[q],[q]}\right)_{[n],\left[n^{\prime}\right]}\right)_{m, m^{\prime}}\right] \\
\approx & \frac{1}{N} \frac{1}{M} \sum_{n=0}^{N-1} \sum_{m=0}^{M-1}\left(\left(\left(\tilde{\mathbf{D}}_{2}^{-1}\right)_{[q],[q]}\right)_{[n],[n]}\right)_{m, m} .
\end{aligned}
$$

Finally, substituting $\tilde{\mathbf{D}}_{2}^{-1}$ from (60) into (61), the final result in Theorem 1 is obtained; this completes the proof.

\section{REFERENCES}

[1] G. D. Surabhi, R. M. Augustine, and A. Chockalingam, "On the diversity of uncoded OTFS modulation in doubly-dispersive channels," IEEE Trans. Wireless Commun., vol. 18, no. 6, pp. 3049-3063, Jun. 2019.

[2] T. Wang, J. G. Proakis, E. Masry, and J. R. Zeidler, "Performance degradation of OFDM systems due to Doppler spreading," IEEE Trans. Wireless Commun., vol. 5, no. 6, pp. 1422-1432, Jun. 2006.

[3] R. Hadani, et al, "Orthogonal time frequency space modulation," in Proc. IEEE WCNC, Mar. 2017, pp. 1-6.

[4] V. Khammammetti and S. K. Mohammed, "OTFS-based multipleaccess in high Doppler and delay spread wireless channels," IEEE Wireless Commun. Lett., vol. 8, no. 2, pp. 528-531, Apr. 2018.

[5] G. Surabhi and A. Chockalingam, "Low-complexity linear equalization for OTFS modulation," IEEE Commun. Lett., vol. 24, no. 2, pp. 330334, Feb. 2020.

[6] S. Tiwari, S. S. Das, and V. Rangamgari, "Low complexity LMMSE receiver for OTFS," IEEE Commun. Lett., vol. 23, no. 12, pp. 22052209, Dec. 2019.

[7] M. K. Ramachandran and A. Chockalingam, "MIMO-OTFS in highdoppler fading channels: Signal detection and channel estimation," in Proc. IEEE GLOBECOM, Dec. 2018, pp. 206-212.

[8] Y. Liu, S. Zhang, F. Gao, J. Ma, and X. Wang, "Uplink-aided high mobility downlink channel estimation over massive MIMO-OTFS system," IEEE J. Sel. Areas Commun., vol. 38, no. 9, pp. 1994-2009, Sept. 2020.

[9] W. Shen, L. Dai, J. An, P. Fan, and R. W. Heath, "Channel estimation for orthogonal time frequency space (OTFS) massive MIMO," IEEE Trans. Signal Process., vol. 67, no. 16, pp. 4204-4217, Aug. 2019.

[10] B. C. Pandey, S. K. Mohammed, P. Raviteja, Y. Hong, and E. Viterbo, "Low complexity precoding and detection in multi-user Massive MIMO OTFS downlink," IEEE Trans. Veh. Technol., Early access, 2021.

[11] S. Li, P. J. Smith, P. A. Dmochowski, H. Tataria, M. Matthaiou, and J. Yin, "Massive MIMO asymptotics for ray-based propagation channels," IEEE Trans. Wireless Commun., vol. 19, no. 6, pp. 39773991, Jun. 2020.

[12] H. Q. Ngo, E. G. Larsson, and T. L. Marzetta, "Aspects of favorable propagation in massive MIMO," in Proc. IEEE EUSIPCO, Sept. 2014, pp. 76-80.

[13] M. Matthaiou, P. J. Smith, H. Q. Ngo, and H. Tataria, "Does massive MIMO fail in Ricean channels?" IEEE Wireless Commun. Lett., vol. 8, no. 1, pp. 61-64, Feb. 2018.

[14] F. Hlawatsch and G. Matz, Wireless Communications Over Rapidly Time-Varying Channels. Academic Press, 2011.

[15] M. Li, S. Zhang, F. Gao, P. Fan, and O. A. Dobre, "A new path division multiple access for the massive MIMO-OTFS networks," IEEE J. Sel. Areas Commun., vol. 39, no. 4, pp. 903-918, Apr. 2021.

[16] A. Chatterjee, V. Rangamgari, S. Tiwari, and S. S. Das, "Non orthogonal multiple access with orthogonal time frequency space signal transmission," arXiv preprint arXiv:2003.06387, 2020.

[17] F. Zhang, Matrix Theory: Basic Results and Techniques. Springer Science \& Business Media, 2011.

[18] P. Singh, A. Gupta, H. B. Mishra, and R. Budhiraja, "Low-complexity ZF/MMSE receivers for MIMO-OTFS systems with imperfect CSI," arXiv preprint arXiv:2010.04057, 2020.

[19] L. Liu, G. Peng, and S. Wei, Massive MIMO Detection Algorithm and VLSI Architecture. Springer, 2019. 